\title{
Lemur Loris Digestive Tract Pathologies (Dissection Study)
}

\author{
L. V. Tkachenko* \\ Altai State Agrarian University, Barnaul, Russia
}

\begin{abstract}
A necropsy study of the Lemur Lori was carried out. Physiologically, Lemurs have a very sensitive gastrointestinal tract. Therefore, these animals almost keep the lead in the incidence of pathologies of the oral cavity, stomach, and intestines. The study was carried out in the dissection room of the Veterinary Medicine Faculty of the Altai State Agrarian University. The object was the animal corpse of a Lemur Loris: animal species: monkey; gender: female; age: 1.5 years; breed: Loris; the owner is a private zoo; provisional diagnosis: not known. The following methods were used: history taking; postmortem examination according to Shor's method with a description of the results obtained; photographing; analysis of the data obtained with the help of fact-finding. As a result of the postmortem examination, the following changes were recorded: pulmonary edema, acute congestive hyperemia and emphysema; acute expansion of the right atrium and ventricle of the heart; acute catarrhal-hemorrhagic esophagitis and gastroenteritis; acute hemorrhagic pancreatitis; acute serous-hemorrhagic lymphadenitis of the mesenteric lymph nodes; liver and kidneys fatty degeneration; cyanosis of the conjunctiva, tunica mucosa of the mouth; obesity. Thus, death occurred as a result of pulmonary arrest (pulmonary edema), which was the result of autointoxication (inflammatory processes in the stomach, intestines, pancreas). The cause of autointoxication is a long-term and major violation of the norms of feeding and maintenance, stress, and presumably a drug that was administered to the animal, namely, an incorrect dose or individual intolerance of its constituent components.
\end{abstract}

\section{Introduction}

In the modern world, exotic animals are increasingly purchased for home keeping, for private animal collections or ethnic parks. At the same time, it is not always possible and sometimes even impossible to reproduce fully the way of life, hierarchical ties, and pair formation.

As a result, animals suffer from various pathologies that lead to their death.

That is why each case of exotic animals death should be studied, and the results of necropsy studies should be accurately described. This will allow veterinary specialists, biologists, zoologists to understand better all the mechanisms of pathology development, and therefore give competent advice to the owners of exotic animals.

*Corresponding author : rabota36@bk.ru 
Lemur Loris is a representative of the Strepsirrhini suborder, the Prosimii suborder, the Lemuridae family, the Lemur genus. Naturalist G. Buffon ranked Lemur Loris among Lemurs [3].

The name of the animal Loris (Stenops) comes from "Loeris", translated from Dutch as "a clown". What is more, the Romans called Lemurs the souls of the dead, who guarded the family and home [16].

They are the arboreal animals, whose historical homeland is Madagascar and the Comoros. It is impossible to take lemurs out of their natural habitats, so they can only be purchased from breeders [2, 3]. Lemurs are listed as endangered species, which are protected in accordance with the Law of the Russian Federation and by different international environmental organizations. Lemur trade is prohibited in Russia and throughout the world $[12,13]$.

Lemurs Loris are sometimes compared to cats. This explains their great popularity among private breeders in any part of the world. It is difficult to reproduce Loris' living conditions at home. In addition to society, cubicles and other things, it is necessary to keep these animals to the diet, on which the quality of Loris' life directly depends $[6,10]$.

In captivity, these animals get used to almost any food [7, 14]. Despite being omnivorous, they have a very sensitive gastrointestinal tract. Consequently, these animals almost keep the lead in the incidence of pathologies of the oral cavity, stomach, and intestines, such as inflammatory processes, bezoars, intestinal obstructions, etc. [14].

Trichobezoars are stones formed due to the accumulation of wool in the stomach of an animal. For their painless removal, some drugs are prescribed, and some vegetables are used as preventive measures $[5,15]$.

In general, Loris' diet in captivity should contain vegetables, seedless fruits, berries, eggs and meat, as well as insects, larvae, plus vitamin complexes. Everything should be pre-treated with hot water. It is strictly forbidden to feed them with any dishes from the table [3].

The average age of lemurs is 8-13 years [3]. But in nature, mortality in the first year of life is $50 \%$, and in zoos this indicator is minimal $[9,6]$. The source $[4,8]$ mentions a high level of stress due to improper maintenance, care and lack of veterinary care, which leads to a rapid death coming.

There are no data available in any literature concerning animal mortality and description of pathological changes in the gastrointestinal tract of Lemurs Loris kept at home.

The aim of our study was to establish the cause of death of the Lemur Loris.

Research objectives are as follows: 1 . To determine the pathological changes of the Lemur Loris.

2. To establish the cause of death of the Lemur Lori.

3. To establish fact-findings between the cause of death and the conditions of maintenance and feeding.

\section{Methodology}

The study was carried out in the dissection room of the Veterinary Medicine Faculty of the Altai State Agrarian University. The internal organs of a deceased Lemur served as the object for the study.

Animal species: monkey

gender: female;

age: 1.5 years;

breed: Loris;

the owner is a private zoo;

provisional diagnosis: not known. 
Methods: 1. History taking [1]. 2. Postmortem examination according to Shor's method with a description of the results obtained $[11,14]$. 3. Macrophotography performed with a Sony camera. 4. Analysis of the data obtained with the help of fact-finding [2].

\section{Results and Discussion}

The history taking showed the following. The animal was kept at home, then, due to its excess weight, it was transferred to a private zoo. After strict diet crossover (vegetables, fruits), with the loss of weight, the Lemur felt unwell sharply. As a result, it was suggested that the stomach was clogged with hair (trichobezoars), and a drug used to remove hair from the cats' stomach was prescribed to solve this problem. Duration of admission made 14 days, after which the animal died. The name of the drug, dosage, frequency rate was not indicated by the owner.

As a result of the necropsy study, the following changes were recorded - an animal of strong proportional constitution, fatty fill (Fig. 1).

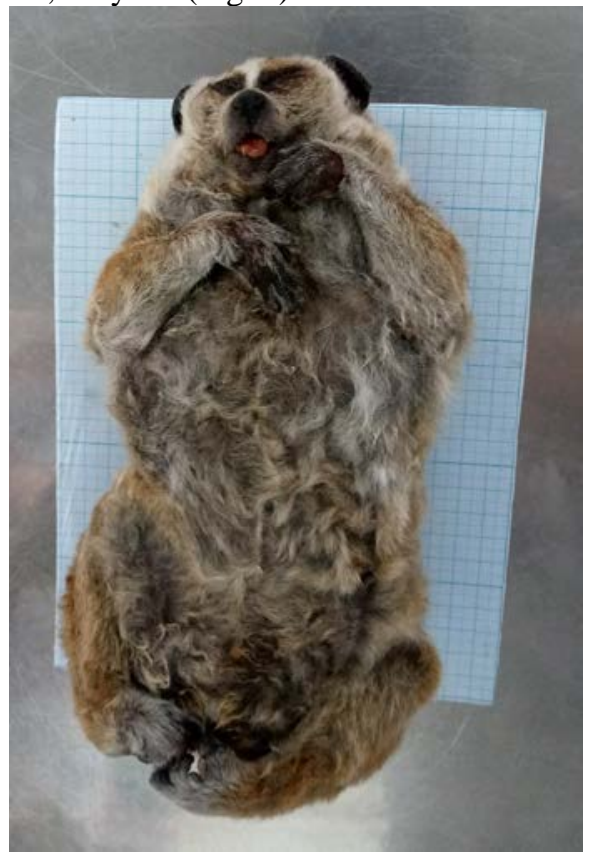

Fig. 1. Lemur Loris. Age - 1.5 years. Gender - female. General form. Strong proportional constitution, fat.

Esophagus: the position is not disturbed, adventitia without overlaps and enlargements, blood vessels highly filled. A small amount of content, consisting of white particles of 0.2$0.3 \mathrm{~mm}$, soft consistency (pieces of bread) (Fig. 2.1.), permeability is not impaired. The mucosa, submucosa, muscular membranes are thickened, rugosity is well expressed, cherry-colored, moist, abundantly covered with mucoid masses of a reddish color (Fig. 2.2.). 


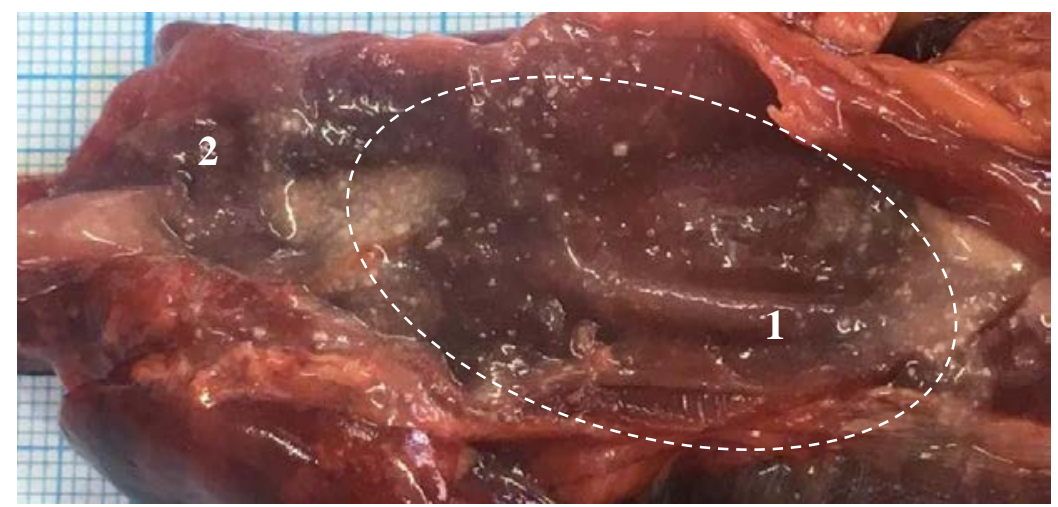

Fig. 2. Lemur Loris. Age - 1.5 years. Gender - female. Esophagus (dissected). 1. A small amount of content: white particles of 0.2-0.3 mm, soft consistency (pieces of bread). 2. Section of acute catarrhal-hemorrhagic esophagitis.

Stomach: the position is not disturbed, the serous membrane without overlaps and enlargements, blood vessels highly filled. No content. Permeability is not impaired. The mucosa, submucosa, muscular membranes are thickened, rugosity is well expressed, redcherry, moist. The surface is abundantly covered with a red mucus mass (Fig. 3).

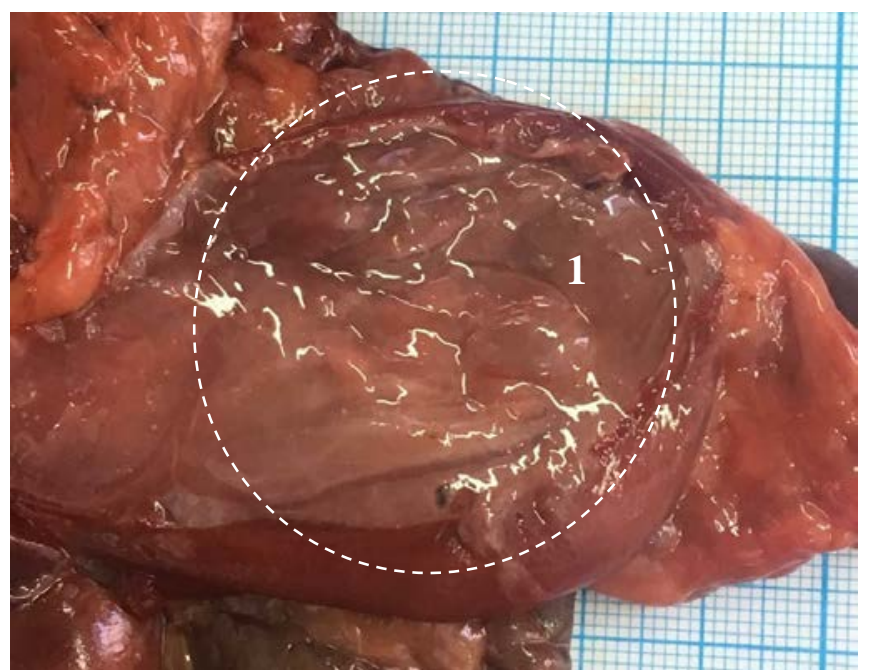

Fig. 3. Lemur Loris. Age - 1.5 years. Gender - female. Stomach (dissected). 1. Section of acute catarrhal-hemorrhagic esophagitis.

Pancreas: the volume is increased, the shape is specific, the surface is slightly bumpy, red-cherry in color with sections of white and yellow color, the consistency is doughy, the structure in the dissection - the pattern is erased (Fig. 4). 


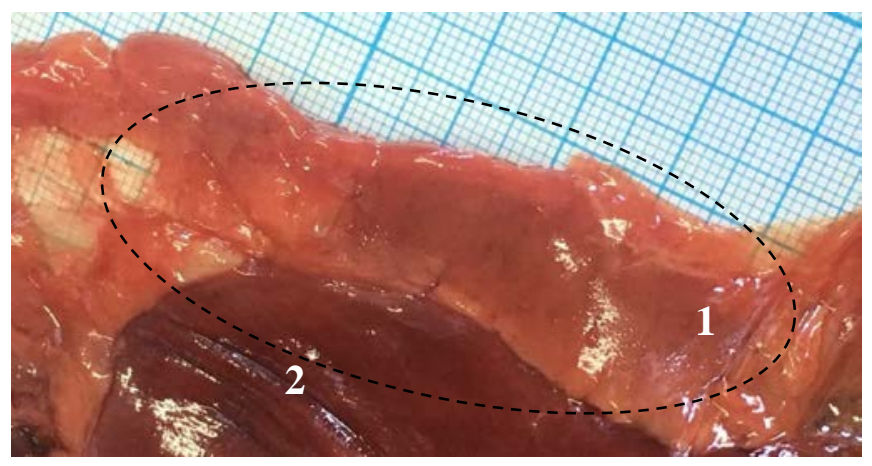

Fig. 4. Lemur Loris. Age - 1.5 years. Gender - female. Stomach (dissected). 1. Section of acute hemorrhagic pancreatitis. 2. Stomach.

Intestines: on the mesentery, there is a large amount of fatty deposits of gray and pink color (Fig. 5.1.), the blood vessels are highly filled with blood. The mesenteric lymph nodes (Fig. 5.3.) are sharply enlarged in volume and elongated, the surface is smooth, red-cherry, of doughy consistency, the structure in the dissection - the pattern is smoothed, red mucuslike mass flows down in the dissection (Fig. 5.2.).

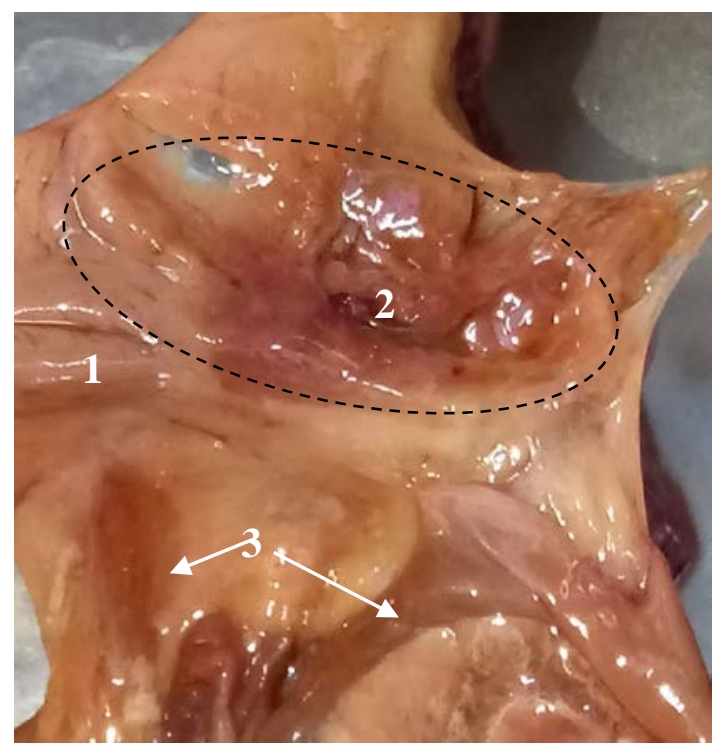

Fig. 5. Lemur Loris. Age - 1.5 years. Gender - female. Mesenteric lymph node (dissected) 1. A large amount of fat deposits. 2. A section of acute serous-hemorrhagic lymphadenitis of the mesenteric lymph nodes. 3. Mesenteric lymph node.

Duodenum: the position is not disturbed, the serous membrane without overlaps and enlargements, the blood vessels highly filled. No content. Permeability is not impaired. The mucosa, submucosa, muscular membranes are thickened, the rugosity is moderately expressed, red-cherry, moist, abundantly covered with red mucus-like mass.

The jejunum: the position is not disturbed, the serous membrane without overlaps and enlargements, the blood vessels highly filled. No content. Permeability is not impaired. The mucosa, submucosa, muscular membranes are thickened, the rugosity is moderately expressed, red-cherry, moist, abundantly covered with red mucus-like mass. 
The ileum: the position is not disturbed, the serous membrane without overlaps and enlargements, the blood vessels highly filled. No content. Permeability is not impaired. The mucosa, submucosa, muscular membranes are thickened, the rugosity is moderately expressed, red-cherry, moist, abundantly covered with red mucus-like mass (Fig. 6).

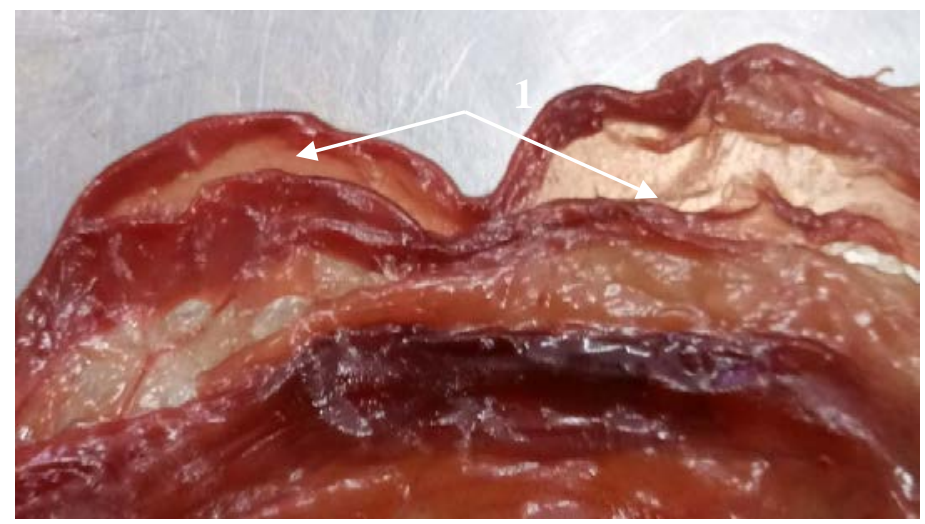

Fig. 6. Lemur Loris. Age - 1.5 years. Gender - female. Small intestine (dissected). 1. Section of acute catarrhal-hemorrhagic enteritis.

Blind intestine: the position is not disturbed, the serous membrane without overlaps and enlargements, the blood vessels highly filled. No content. Permeability is not impaired. The mucosa, submucosa, muscular membranes are thickened, the rugosity is moderately expressed, red-cherry, moist, abundantly covered with red grout.

Segmented intestine: the position is not disturbed, the serous membrane without overlaps and enlargements, the blood vessels highly filled. No content. Permeability is not impaired. The mucosa, submucosa, muscular membranes are thickened, the rugosity is moderately expressed, red-cherry, moist, abundantly covered with red grout.

Straight intestine: the position is not disturbed, the serous membrane without overlaps and enlargements, the blood vessels highly filled. No content. Permeability is not impaired. The mucosa, submucosa, muscular membranes are thickened, the rugosity is moderately expressed, red-cherry, moist, abundantly covered with red grout.

Liver: the volume is increased, of a specific shape, smooth surface, red-brown, sloppy texture, the structure in the dissection is slightly smoothed. Scraping contains ample drops of fat

Gallbladder: the position is not disturbed, the serous membrane without overlaps and enlargements, the blood vessels highly filled. Content: homogeneous, moderate amount, liquid consistency, green color, specific odor. Permeability is not impaired. Mucous, fibromuscular, sub-serous base, serous membrane are not thickened, the rugosity is not pronounced, greenish-yellowish in color, moist, without overlaps.

Kidneys: the volume is increased, of a specific shape, smooth surface, red-brown, sloppy texture, the structure in the dissection bulges out, slightly smoothed. Scraping contains ample drops.

In general, the following diagnoses were established based on the results of the necropsy study:

1. Pulmonary edema, acute congestive hyperemia and emphysema.

2. Acute expansion of the right atrium and ventricle of the heart.

3. Acute catarrhal-hemorrhagic esophagitis, gastroenteritis.

4. Acute hemorrhagic pancreatitis.

5. Acute serous-hemorrhagic lymphadenitis of the mesenteric lymph nodes.

6. Liver and kidneys fatty degeneration. 
7. Cyanosis of the conjunctiva, tunica mucosa of the mouth.

8. Obesity.

\section{Conclusion}

Thus, having analyzed the information obtained, we conclude that the death of the animal occurred as a result of pulmonary arrest (pulmonary edema), which was the result of autointoxication (inflammatory processes in the stomach, intestines, pancreas).

In our opinion, the cause of autointoxication is a long-term and major violation of the norms of feeding and maintenance, stress, and presumably a drug that was administered to the animal, namely, an incorrect dose or individual intolerance of its constituent components.

\section{References}

1. Anamnesis. - URL: https://big_medicine.academic.ru/1583/ ANAMNESIS (Accessed on 2.04.2021).

2. Bolshakov O.P. Didactic and ethical aspects of research on biomodels and laboratory animals. O.P. Bolshakov, N.G. Neznanov, R.V. Babakhanyan. High-quality clinical practice. - 2002. - No. 1. - pp. 58-61.

3. Bram A.E. Life of animals. M.: Terra, 1992. pp. 135-143.

4. Types of Lemur Lori. - URL: http://zhivotnue.ru/index_ru.php?cat=zhivotnue_krasnoi_knigi\&obl=lyamyr_lori\&ind= 60 (Accessed on 2.04.2021).

5. Davenport G.M. Method and composition for regulation of wool excretion with feces and prevention of trichobezoar formation. G.M. Davenport, G.D. Sunwold, G.A.

Reinhart, M.G. Hake. Patent for invention - RU 2250776 C2, 27.04.2005. - Application No. 2002120921/15 dated 05.01.2001.

6. Ten unexpected facts about lemurs. - URL: https://fishki.net/2619609-10neozhidannyh-faktov-o-Lemurah.html (Accessed on 2.04.2021).

7. Animal life. V.7. Mammals. Ed. by V.E. Sokolov. - 2nd ed., Rev. - M.: Education, 1989. - pp. 122-129.

8. Lemur Lori. - URL: https://simple-fauna.ru/wild-animals/Lemur-lori (Accessed on 2.04.2021).

9. Small and large fat Lori. - - URL:https://chins.ru/showthread.php/203-ЛемурыЛори/page19 (accessed on 2.04.2021).

10. Meshik V.A. Formation of pairs and groups of primates in the zoo .V.A. Meshik. Research in zoological parks. - 2014. - No. 30. - pp. 161-205.

11. Pathological autopsy of animals. Training for private veterinarians, 2013. - URL: http://vsb.kg/uploads/Old_Modul/Руководство\%20для\%20участника_Патан_Роксан a.pdf (Accessed on 2.04.2021).

12. Prokhorov V.G. Wildlife samples smuggling: Contemporary history .V.G. Prokhorov. Eurasian Law Journal. - 2009. - No. 12 (19). - pp. 95-99.

13. Rare animals from the Red Book of Russia and the whole world. - URL: https://simplefauna.ru/animals-life/redkie-zhivotnye-iz-krasnoj-knigi (Accessed on 2.04.2021). 
14. Tkacheva E.Yu. Possibilities of non-contact monitoring of the reproductive state and stress level of zoo animals by methods of hormonal analysis. E.Yu. Tkacheva, O.B. Lifanova. Scientific research in zoological parks. - 1998. - No. 10 (10). - pp. 236-245.

15. Trichobezoars - wool in the stomach. - URL: http://lorises.ru/zdorovie.html (Accessed on 2.04.2021).

16. R. Mittermeier, J. Ganzhorn, W. Konstant, K. Glander, I. Tattersall, C. Groves, A. Rylands, A. Hapke, J. Ratsimbazafy, M. Mayor, E. Louis jr., Y. Rumpler, C. Schwitzer und R. Rasoloarison: Lemur Diversity in Madagascar. In: International Journal of Primatology 29 (2008), pp. 1607-1656. 